\title{
The Analysis of the Cannon Gun Tube Wearing Life Based on Random Using Condition
}

\author{
Tian-Xiao CUI, Jie LI, Guang-Sheng LIU, Chang-Zhi JIA a, \\ Mechanical Engineering College, No.77, Heiping West Road, Xinhua District, Shjiazhuang, China \\ ajiachangzhi@126.com \\ *Corresponding author
}

Keywords: Barrel Life, Ablation Wear, Continuous Fire, Highest Wall Temperature, Radial Ablation Wear.

\begin{abstract}
In view of the problem that the artillery tube life is difficult to accurately forecast under the random using condition, through the analysis of the heat transfer process of the inner wall of the tube body, introducing internal heat strengthening hypothesis, the calculation method of the highest inner wall temperature is obtained and its the variation regularity of ablation wear and the maximum wall temperature is studied. At the same time, the wear calculation method comes up when artillery is shooting continuously under the circumstance of uncertain gunpowder temperature ,uncertain shooting time interval and uncertain propellant charge.
\end{abstract}

\section{Introduction}

In the filed of large diameter $(\geq 100 \mathrm{~mm}$ ) artillery design, in order to enhance the artillery power, it has to have higher velocity and firing rate.As the result, it gives rise to more aggravated erosion and wear of cannon gun tube wall inevitably.Cannon gun tube status affects the result of the projectile hit the target directly, and the barrel life determines the life of the artillery systems in a certain extent. Therefore, the test and analysis on the problem of barrel life is of great significance.

In view of the random using conditional random problems in engineering application, this paper studies and analyses the barrel inner wall wear analysis model under the condition of continuous fire, provides the scientific basis for the research of dynamic barrel life test and analysis .

\section{Tube Wall Erosion Wear Calculation}

Studies have shown that cannon gun tube erosion wear is mainly caused by three aspects: mechanical abrasion, chemical reaction and thermal ablation[1,2]. Scholars usually analysis the barrel life from these three factors. However, these three factors are not independent. this uncertainty coupling relationship among them.

\section{Life Loss Matrixing under Gunpowder Changing}

Domestic and international scholars summed up the equivalent full charge coefficient conversion method which can more accurately calculate the barrel life loss situation under the different shooting conditions (charge, projectile type) through analysis a large number of experimental laws[3]. After a large number of experiment statistics and data analysis, the national artillery equivalent loading coefficient conversion method is summarized as below:

$$
k=\left(p_{m}^{\prime} / p_{m}\right)^{1.4}\left(v_{0}^{\prime} / v_{0}\right)
$$

Where: $k$--conversion coefficient

$p_{m}$-- the maximum bore pressure under full charge

$v_{0}$-- the initial muzzle velocity under full charge

$p_{m}^{\prime}$--the maximum pressure of a converted charge 


\section{$v_{0}^{\prime}$--the initial muzzle velocity of a converted charge}

\section{Abrasion Quantity Calculation under Single Shell Condition}

With the development of research about cannon gun tube erosion wear mechanism, it was found that the heating effect caused by the gunpowder gas is caused the main factor which leads to the phenomenon of tube wall erosion and wear.Many scholars have made a lot of research work and get a lot of practical experience calculation formulas on body tube wall erosion and wear[4]. At present,the body experience formula for tube wall erosion wear that is highly recognized is:

$$
w=A e^{B T_{w}}
$$

Where: $w$-- barrel wear loss(um)

$$
\begin{aligned}
& A 、 B \text {-- empirical constant } \\
& T_{w} \text {--maximum temperature of inner tube face }(\mathrm{k})
\end{aligned}
$$

Eempirical constants A and B is determined by the cannon gun tube wall shape, size, material and powder properties, etc. And this formula is generally recognized by the domestic and foreign experts, and the room-temperature body tube inner surface temperature empirical formula and continuous shooting body tube inner surface temperature empirical formula are respectively established after further research.

$$
\begin{aligned}
& T_{w}=1.09\left(\frac{T_{f}-\Delta T_{c}-600}{d}\right)\left(\omega p_{m}\right)^{0.5} \\
& T_{w}^{N}=0.4632\left(T_{f}-\Delta T_{c}-600\right) \omega^{0.75}(N-1)^{0.5} \frac{R^{0.5}}{d^{0.5}}
\end{aligned}
$$

Where: $T_{f}$-- gunpowder explosion temperature $(\mathrm{k})$

$\Delta T_{c}$--additive temperature correction $(\mathrm{k})$

$d$--bore diameter (mm)

$\omega$--charge $(\mathrm{Kg})$

$p_{m}$-- the maximum bore pressure (MPa)

$N$--the actual number of projectile

$T_{w}^{N}$--the highest temperature of tube inner face after shooting $N$ shells

$R$--the actual rate $\left(\mathrm{min}^{-1}\right)$

\section{Analysis of Inner Wall Temperature}

\section{Inner Wall Heat Reinforcement Hypothesis}

According to the study, duo to the time series of artillery projectile and the charging conditions are random, accurate calculation on the impact of each shot caused by the bore inner wall wear and tear necessary for the accurate assessment of the gun barrel life. And there is the a specific law between the burning erosion and the maximum temperature of the inner wall .

In view of the random artillery projectile and random charge, barrel temperature field distribution is very complex under the continuous shooting condition and it is very difficult to establish the maximum temperature calculation model of inner tube wall accurately. In order to obtain the maximum temperature inside the tube, a lot of analysis has been done and the effect is 
gradually accumulated from the fist projectile to the Nth projectile. And the relationship is shown as fellow.

$$
T_{N}^{\prime}=T_{N}+h_{N-1}\left(t_{N-1}\right) \cdot T_{N-1}^{\prime}
$$

Where : $T_{N}^{\prime}$--the maximum temperature of the inner wall of the tube after continuous shot $T_{N}$--the maximum temperature of the inner wall of the first projectile in the case of cold gun

$h_{N-1}\left(t_{N-1}\right)$--the correction function after the $(N-1)$ th shot

$t_{N-1}$--the time interval between the previous projectile and the Nth projectile

$h_{N-1}\left(t_{N-1}\right)$ is related to the number of shooting, time and artillery structure and other. In the formula (5), to calculate the maximum temperature of the inner wall of the borehole during continuous shooting $\mathrm{N}$ times, it is necessary to calculate the maximum temperature of the inner wall of the gun after the first shot as the second base of influence, and the first and second time of the projectile interval as the second impact coefficient. Calculate the maximum temperature of the inner wall in the case of the second cold gun. Both are superimposed as the second highest temperature of the inner wall; The maximum temperature of the second inner wall is used as the base of the next influence, and the second and next time the projectile interval as the next impact coefficient. Calculate the maximum temperature of the inner wall in the case of the next cold gun. Both are added as the next highest temperature of the inner wall and so on, until the Nth.

As can be seen, consider the effect of the former N-1 times on the Nth time, that the former N-1 times on the Nth role is independent of each other, as follows

$$
T_{N}^{\prime}=T_{N}+\sum_{i=2}^{N} h\left(t_{i-1}\right) T_{i-1}
$$

Where: ${ }^{T_{i-1}}$--In the case of cold guns, the maximum temperature of the i-1 projectile inner wall

$$
\begin{aligned}
& h\left(t_{i-1}\right) \text {--Time-dependent correction function } \\
& t_{i-1} \text {--The time interval between the } \mathrm{i}-1 \text { and the } \mathrm{N} \text {-th projectile }
\end{aligned}
$$

Equation (6) Relative formula (5) simplifies the calculation, it is only necessary to calculate the time of correction of the maximum temperature of the inner wall of the borehole and the time correction function within a certain range after the shotgun. It is possible to reduce the error due to successive coupling.

\section{Time Correction of The Temperature of The Inner Wall}

Through the analysis of the maximum temperature time curve of each inner wall, can be found in the inner wall of the highest temperature basically meet the following rules: After 10 seconds, the maximum temperature of the inner wall reduced to about $20 \%$ of the initial value; 50 seconds after the maximum temperature of the inner wall reduced to about $10 \%$ of the initial value; After 1000 seconds, the maximum temperature of the inner wall to reduce the ambient temperature. And in the above three time the process of temperature changes in line with a certain linear requirements. According to the application of interpolation method can simplify the body wall of the maximum temperature function as follows 


$$
T=\left\{\begin{array}{cc}
T_{w}(1-0.08 t) & (t \leq 10) \\
0.2 T_{w}[1-0.0125(t-10)] & (10 \leq t \leq 50) \\
0.1 T_{w}[1-0.00105(t-50)] & (50 \leq t \leq 1000)
\end{array}\right.
$$

Where: $T$--The maximum temperature required

$T_{w--T h e}$ maximum temperature of each shot

It should be emphasized that the temperature unit in this type is ${ }^{\circ} \mathrm{C}$, and the minimum temperature of the inner wall is the ambient temperature.

\section{The Prediction Model of Pipe Body Life}

The life model of each gun is different. In the following, taking a certain type of gun as the object of study, the loss law of its tube life is studied.

\section{The Relationship between The Maximum Bore Temperature and The Inner Wall}

The caliber of a certain gun is $130 \mathrm{~mm}$, the design life is 1000 times, and it is designed for the divided drug. There are 5 kinds of drug numbers, and the charge design is shown in Table 1.

Table 1. Design of a certain gun charge

\begin{tabular}{lccccc}
\hline Charge number\# & 0 & 1 & 2 & 3 & 4 \\
\hline Charge quantity $(\omega)$ & 12.9 & 11 & 6.5 & 5.22 & 3.92 \\
Bore pressure $\left(\mathrm{p}_{\mathrm{m}}\right)$ & 315 & 300 & 270 & 156.8 & 110 \\
Initial velocity $\left(v_{0}\right)$ & 930 & 810 & 705 & 620 & 525
\end{tabular}

According to the formula (1) and the formula (3), the equivalent total charge conversion coefficient and the maximum temperature of the inner wall can be calculated respectively when the ambient temperature is 15 DEG C; At the same time, when the average bore wear loss of the cannon barrel reaches $3.5 \%$ of the caliber, it is considered the end of life, and the amount of wear and the expected life of each type of propellant in the gun can be calculated, as shown in Table 2.

Table 2. Bore temperature, wear rate and life of each charge

\begin{tabular}{llllll}
\hline$T_{w}$ & 1282.6 & 1155.8 & 842.9 & 575.6 & 417.8 \\
$w$ & 4.550 & 3.702 & 2.178 & 1.142 & 0.540 \\
$N$ & 1000 & 1229 & 1636 & 3982 & 7728
\end{tabular}

Draw the curve from the corresponding data of the wall temperature and radial wear of some gun in Table 2, and fit it as shown in the following Fig.1.

For example, the relation between the maximum temperature of the interior wall and the amount of wear is shown, the black solid line is the fitted polynomial curve, and the red dashed line is exponential curve. It is obvious that the polynomial curve is better than the exponential curve. Therefore, the relation between the maximum bore temperature and the corresponding ablation wear quantity of a gun is established.

$$
w=2 \times 10^{-6} T_{w}^{2}+10^{-3} T_{w}-0.236
$$


Where: $w$--wear volume (um)

$T_{w}$--the highest temperature of bore $\left({ }^{\circ} \mathrm{C}\right)$

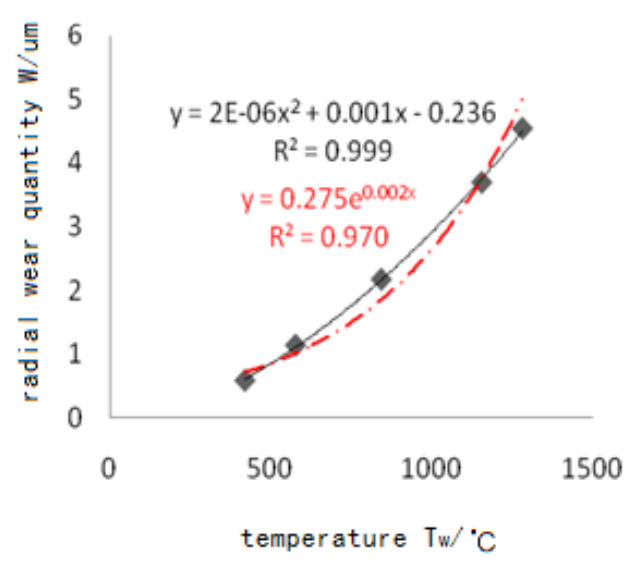

Fig. 1. Fitting relationship between maximum temperature and wear volume of inner wall

\section{The Body Tube Life Prediction Model}

From section 3.1, cannon gun is judged failure when the wear loss (4550 um) is up to $3.5 \%$ of diameter.

The ablation wear of the cannon gun will successively accumulate if resume firing is executed, and barrel ballistic performance will reduce gradually. So the body tube life prediction model is established as follows:

$$
I=\left(1-\sum_{i=1} \frac{w_{i}}{4550}\right) \times 100 \%
$$

Where: $I$--tube reserved life

$$
w_{i} \text {--the radial wear volume of each fire }
$$

When $I$ reduces to 0 , the cannon life is indicated as termination. The $I \times 100$ represents the room temperature gun life number under the condition of residual standard gunpowder temperature and full charging .

After a certain time of use, the cannon gun tube wall has certain radial wear. At this point, the using units of this artillery can arrange maintenance and shooting schedule based on the residual life of artillery gun tube. For example, a certain type of cannon requires one overhaul repairing and 4 times medium repairing during its whole life. The standard $I$ can be defined as 50\%, 16.6\%,33.3\%, $66.9 \%$ and $83.6 \%$ respectively for the overhaul repairing and medium repairing. Also, the army need to arranges the preparation for combat or training on the basis of body tube residual life.

\section{Conclusion}

In this paper, the rule of heat transfer and rise of the chamber body tube wall temperature was studied and tube life prediction under the random using random condition is achieved. And get the following conclusion:

(1) The problem of calculation of pipe wall wear under diverse conditions in the actual using process of the gun.

(2) This paper made some assumptions and approximations to simplify the highest body tube wall temperature calculation, but this method still need further study. 
(3) Considering the body tube life determines the whole life of the cannon basically, this article established the body tube life calculation model. If this model is applied to real life prediction of artillery, the cannon gun tube technology condition can be quickly grasped.

\section{Acknowledgements}

This work was financially supported by the Hebei Province Science and Technology Project (15210701D).

\section{References}

[1] Weidong Song, Chenli Tao. Cannon gun tube residual life evaluation method discussion [J]. Journal of testing technology, 2002, 16:163-166.

[2] Ian a. ohnston. Understanding and Prediction Gun Barrel Erosion [R]. Technical Report DSTO TR - 1757, 2005.

[3] Lianrong Wang, Peiqin Zhang. Gun interior ballistic calculation handbook [M]. Beijing: national defence industry press, 1987:74-77.

[4] Qing OuYang. Cannon gun tube erosion wear and life problem research [D]. Nanjing: nanjing university of science and technology, 2013. 